\title{
Efeitos tóxicos de plantas medicinais comercializadas in natura no Município de São
}

\section{Luís/MA: uma revisão de literatura}

Toxic effects of medicinal plants commercialized in natura in São Luís /MA: Aliterature review

Efectos tóxicos de las plantas medicinales comercializadas en natura en São Luís /MA: una revisión de la literatura

Recebido: 08/04/2021 | Revisado: 15/04/2021 | Aceito: 03/05/2021 | Publicado: 16/05/2021

\author{
Elane de Oliveira Gatinho Vieira \\ ORCID: https://orcid.org/0000-0002-8504-4728 \\ Instituto Florence de Ensino Superior, Brasil \\ E-mail: elanefarmaceutica@yahoo.com \\ Raquel Maria Trindade Fernandes \\ ORCID: https://orcid.org/0000-0003-0345-5556 \\ Universidade Estadual do Maranhão, Brasil \\ E-mail: raquelfernandes@professor.uema.br
}

\begin{abstract}
Resumo
Objetivos: Objetivou-se realizar uma revisão bibliográfica a respeito de dez plantas medicinais que apresentam potencial tóxico frequentemente comercializadas na forma in natura em São Luís-MA e um catálogo ilustrativo sobre as principais espécies comercializadas. Método: Foram selecionados artigos sobre o tema, sobretudo, por meio dos bancos de dados LILACS, SCOPUS e PUBMED, utilizando como descritores as seguintes palavras: "plantas medicinais", "toxicidade de drogas" e "fitoterapia" e seus respectivos termos em inglês. Foram utilizadas 43 referências bibliográficas. Resultados: No Maranhão, o uso de plantas como recurso terapêutico é uma prática que vem passando de geração a geração. Entre as plantas mais comercializadas se encontravam a babosa, mastruz, hortelã, boldo, erva-doce, quebra-pedra, arruda, malva-santa, manjericão, pata-de-vaca. Destaca-se que todas possuem alguma característica quanto à toxicidade em consumos de grandes quantidades, entretanto estudos da literatura demonstram que na maioria das vezes a população desconhece esses efeitos. Conclusões: É indiscutível o potencial terapêutico existente nas espécies vegetais brasileiras, sendo as plantas medicinais muito utilizadas no Brasil para o tratamento e prevenção de doenças. Para tanto, faz-se necessário o delineamento e execução de protocolos adequados que viabilizem seu uso e um maior esclarecimento à população sobre os efeitos tóxicos das mesmas.
\end{abstract}

Palavras-chave: Plantas medicinais; Toxicidade de drogas; Fitoterapia.

\begin{abstract}
Objectives: The objective was to carry out a bibliographic review about ten medicinal plants that have toxic potential frequently sold in natura form in São Luís-MA and an illustrative catalog on the main species sold. Method: Articles on the topic were published, mainly through the LILACS, SCOPUS and PUBMED databases, using the following words as descriptors: "medicinal plants", "drug toxicity" and " herbal medicine "and its respective terms in English. 43 bibliographical references were used. Results: In Maranhão, the use of plants as a therapeutic resource is a practice that has been passed down from generation to generation. Among the most commercialized plants were aloe vera, mastruz, mint, bilberry, fennel, stone breaker, rue, hollyhock, basil, paw-of-cow. It is noteworthy that all of them have some characteristic regarding the toxicity in consumption of large quantities, however studies in the literature demonstrate that in most cases the population is unaware of these effects. Conclusions: The therapeutic potential of Brazilian plant species is indisputable, and medicinal plants are widely used in Brazil for the treatment and prevention of diseases. To this end, it is necessary to design and execute adequate protocols that make their use feasible and provide greater clarification to the population about their toxic effects.
\end{abstract}

Keywords: Medicinal Plants; Drug-Related Side Effects and Adverse Reaction; Phytotherapy.

\section{Resumen:}

Objetivos: El objetivo fue realizar una revisión bibliográfica sobre diez plantas medicinales que tienen potencial tóxico frecuentemente vendidas en forma natural en São Luís-MA y un catálogo ilustrativo de las principales especies vendidas. Método: Se publicaron artículos sobre el tema, principalmente a través de las bases de datos LILACS, SCOPUS y PUBMED, utilizando como descriptores las siguientes palabras: "plantas medicinales", "toxicidad de fármacos" y "fitoterapia" y sus respectivos términos. en Inglés. Se utilizaron 43 referencias bibliográficas. Resultados: En Maranhão, el uso de plantas como recurso terapéutico es una práctica que se ha transmitido de generación en generación. Entre las plantas más comercializadas se encuentran el aloe vera, mastruz, menta, arándano, hinojo, 
picapedrero, ruda, malvarrosa, albahaca, pata de vaca. Es de destacar que todos ellos tienen alguna característica en cuanto a la toxicidad en el consumo de grandes cantidades, sin embargo los estudios en la literatura demuestran que en la mayoría de los casos la población desconoce estos efectos. Conclusiones: El potencial terapéutico de las especies vegetales brasileñas es indiscutible y las plantas medicinales son ampliamente utilizadas en Brasil para el tratamiento y prevención de enfermedades. Para ello, es necesario diseñar y ejecutar protocolos adecuados que viabilicen su uso y brinden mayor claridad a la población sobre sus efectos tóxicos.

Palabras clave: Plantas medicinales; Efectos Colaterales y Reacciones Adversas Relacionados con Medicamentos; Fitoterapia.

\section{Introdução}

O uso de plantas com atividade farmacológica pelo ser humano teve início na idade antiga. A terapêtica dos dias atuais muito se deve ao auxílio de plantas medicinais no desenvolvimento direto ou indireto de medicamentos. Inúmeras pesquisas publicadas nos últimos anos reafirmam a importância dos produtos naturais como fonte de fármacos, entre estes pode-se citar a morfina, pilocarpina, digitálicos etc. (Barreiro \& Bolzani, 2009) (Valadas et al., 2021).

Os primeiros relatos sobre plantas medicinais foram feitos em papiro séculos atrás na dinastia do Egito antigo. Esse papiro, encontrado nas proximidades da casa mortuária de Ramsés II, relatou cerca de aproximadamente 100 doenças e um grande número de drogas da natureza animal, vegetal ou mineral. Provavelmente esse foi o primeiro material escrito sobre produtos naturais na cura de doenças. Na Grécia Hipócrates e Teofrasto fizeram os primeiros registros sobre plantas, descrevendo a espécie Papaver somniferum, planta cujo princípio ativo é a morfina. Além disso, evidências arqueológicas mostram que o uso de drogas vegetais era amplo em culturas antigas (Cunha, 2012).

Entretanto não apenas de propriedades benéficas se caracterizam as plantas com atividade farmacológica, algumas espécies produzem características prejudiciais à saúde humana, podendo ser utilizadas para casos de envenenamento intencional, abortos, etc. Muitas destas plantas possuem seus princípios ativos e efeitos maléficos estudados, mas eventualmente novos efeitos desconhecidos surgem que podem causar diversos danos (Argenta et al., 2011).

A importância do grupo das plantas tóxicas, não está só nos riscos que estas representam, mas também nos benefícios que pode proporcionar, quando the é dado um uso adequado. Muitos dos componentes químicos encontrados na farmacologia, são produzidos a partir dessas plantas e uma grande quantidade dos vegetais ou suas partes estão representados em infusões, ungüentos e macerados empregados na medicina tradicional (Prashar et al., 2003).

As plantas medicinais representam a principal matéria médica utilizada pelas chamadas medicinas tradicionais, ou não ocidentais, em suas práticas terapêuticas, sendo a medicina popular a que utiliza o maior número de espécies diferentes. Devido a este fator, as plantas medicinais continuam ocupando lugar de destaque no arsenal terapêutico (Matos, 1998).

O uso de plantas com fins medicinais é uma prática comum no Brasil, passado de geração em geração, seja para tratamento ou prevenção de doenças. Entre as plantas mais estudadas encontram-se a copaíba, alecrim-pimenta, eucalipto, malva, aroeira, entre outras (Nascimento et al., 2007).

$\mathrm{O}$ estado do Maranhão possui grande destaque tratando-se de plantas medicinais. Embora a Fitoterapia atualmente tenha conquistado uma maior visibilidade no âmbito das ações de saúde, ainda existem grandes dificuldades devido a falta de recursos e a questão de patentes. Em especial, a Universidade Federal do Maranhão destaca-se com o herbário Ático Seabra, este vinculado ao Departamento de Farmácia, sendo liderado pela Dra Terezinha Rêgo, contando com mais de 10.800 de espécies de plantas nativas. Vale ressaltar que o herbário possui grande visibilidade e parcerias internacionais, o que valoriza o potencial de Fitoterápicos no estado (Gorberg \& Fridman, 2006).

Objetivou-se realizar uma revisão bibliográfica a respeito de algumas plantas medicinais que apresentam potencial tóxico e um catálogo ilustrativo sobre as principais espécies comercializadas, para o mesmo ter posterior distribuição nas Farmácias do munícipio de São Luís-MA. 


\section{Metodologia}

Nesse estudo foi realizada uma revisão de literatura sobre os efeitos tóxicos de plantas medicinais in natura frequentemente comercializadas nas farmácias de São Luís-MA. Previamente realizou-se uma busca ativa em dez estabelecimentos para assim identificar as plantas mais comercializadas. Para isso, foram selecionados artigos sobre o tema, sem restrição de data, utilizando os bancos de dados PUBMED, SCOPUS e LILACS, e tendo como descritores as seguintes palavras: "plantas medicinais", "toxicidade de drogas" e "fitoterapia" e seus respectivos termos em inglês. Foram selecionadas 43 referências, entre artigos científicos, monografias e livros. Foram selecionados artigos publicados nos idiomas inglês e português, na forma de artigos de pesquisa clínica, de revisão bibliográfica e epidemiológicos.

\section{Revisão de literatura}

No Quadro 1 encontra-se a síntese dos principais achados relacionados a toxicidade das plantas medicinais nos artigos encontrados.

Quadro 1: Relação entre dez das plantas medicinais bastante comercializadas de São Luís-MA e seus efeitos tóxicos.

\begin{tabular}{|c|c|}
\hline PLANTAS MEDICINAIS & EFEITOS TÓXICOS (DOSES ELEVADAS) \\
\hline Arruda (Ruta graveolens) & $\begin{array}{l}\text { Embriotóxica, teratogênica, abortiva, hemorragias, irritação } \\
\text { da mucosa bucal e inflamações epidérmicas (Veiga Junior et } \\
\text { al., 2005) (Rodrigues et al., 2011) (Bochner et al., 2012). }\end{array}$ \\
\hline Babosa (Aloe vera) & $\begin{array}{l}\text { Pode ser citotóxica, hepatotóxica, cólicas, náuseas e diarréias } \\
\text { (Ramos \& Pimentel, 2011). }\end{array}$ \\
\hline Boldo (Peumus boldus) & $\begin{array}{l}\text { Teratogenia, dermatite alérgica, convulsões, hepatotoxicidade } \\
\text { e aborto (Almeida et al., 2000) (Torres et al., 2005). }\end{array}$ \\
\hline Erva doce (Pimpinella anisum) & $\begin{array}{l}\text { Broncodilatação, aumento da produção de estrógeno e } \\
\text { alergias, tumores e convulsões (Mello, Langeloh \& Mello, } \\
\text { 2007) (Santos, 2014). }\end{array}$ \\
\hline Hortelã (Mentha piperita) & $\begin{array}{l}\text { Dispnéia e asfixia, especialmente em crianças e lactentes } \\
\text { (Rodrigues et al., 2011). }\end{array}$ \\
\hline Malva santa (Plectranthus barbatus andr.) & $\begin{array}{l}\text { Hepatotoxicidade, abortos, carcinogenia e nefrotoxicidade } \\
\text { (Costa, 2006). }\end{array}$ \\
\hline Manjericão (Ocimum basilicum) & $\begin{array}{l}\text { Citotoxicidade, convulsões, distúrbios sensoriais e até } \\
\text { psíquicos (Cavalini et al., 2005; Guez, 2014). }\end{array}$ \\
\hline Mastruz (Chenopodium ambrosioides) & $\begin{array}{l}\text { Citotoxicidade, hepatotoxicidade, tremores, irritabilidade, } \\
\text { resposta ao toque, resposta a cauda de aperto, córnea, } \\
\text { convulsões, lacrimejamento, hipotermia, entre outros (Pereira } \\
\text { et al., 2010; Valerio et al., 2015). }\end{array}$ \\
\hline Quebra pedra (Phyllanthus sp.) & Aborto (Rodrigues et al., 2011). \\
\hline Pata de Vaca (Bauhinia forficata l.) & $\begin{array}{l}\text { Aumento dos níveis de ALT e fosfatase alcalina e possível } \\
\text { toxicidade hepática (Peixoto et al., 2012). }\end{array}$ \\
\hline
\end{tabular}

Fonte: Autores.

\subsection{Arruda (Ruta graveolens)}

A espécie Ruta graveolens L. (R. graveolens) é uma planta que pertence à família Rutaceae e tem sido objeto de estudo por diversos pesquisadores devido ao seu efeito antifúngico. Popularmente a R. graveolens é conhecida como arruda, se caracterizando por ser um arbusto pequeno, de origem Mediterrânea, existente em todo o mundo (Reis et al., 2015). 
A $R$. graveolens é utilizada com finalidades medicinais desde a época da civilização grega e romana, se caracterizando por ter diversos compostos que vão proporcionar a atividade medicinal à planta. Esses compostos são os responsáveis pelas diversas atividades farmacológicas, especialmente em processos infecciosos e inflamatórios, patologias digestivas, musculares e tumorais (Mena et al., 2016).

A literatura aponta que a $R$. graveolens possui atividade anti-parasitária, anti-hemorrágica, abortiva, antiespamódica, etc. Tem indicação também para reumatismos e hipertensão, devido às suas propriedades estimulantes. Entretanto, plantas medicinais podem ser potencialmente perigosas, tratando-se da arruda, em doses elevadas pode provocar aborto, fortes hemorragias, irritação da mucosa bucal e inflamações epidérmicas. Plantas como a arruda, que já possuem toxicidade reconhecida correm maiores riscos de erros de administração e uso irracional (Rodrigues et al., 2011; Bochner et al., 2012).

A $R$. graveolens tem contra-indicação principalmente durante a gravidez, pois seu uso acaba exercendo fortes contrações no útero. Além disso, diversos são os estudos que comprovaram o efeito embriotóxico e teratogênico a partir do extrato aquoso das folhas da mesma (Rodrigues et al., 2011).

\subsection{Babosa (Aloe Vera)}

A Aloe vera $(L)$ burm. f. é uma planta pertencente à família Aloaceae, utilizada medicinalmente há muitos anos. É uma planta do tipo herbácea que se caracteriza por crescer em qualquer tipo de solo e não necessita de muito suprimento hídrico. A Aloe vera é popularmente chamada de babosa, aloe, aloe-de-barbados e aloe-de-curaçao. O nome babosa foi dado devido à consistência viscosa (baba) da mucilagem de suas folhas, contendo esta várias substâncias com propriedades ativas na planta (Freitas, Rodrigues \& Gaspi, 2014).

Os primeiros relatos sobre a Aloe vera foram na Mesopotâmia 2100 a.C. Seu nome se origina do idioma árabe, que significa amarga e brilhante. Também ganhou notoriedade no Egito antigo, por suas propriedades benéficas na pele e cabelo. Desde 1932 foi reconhecida pela Farmacopéia Britânica como droga oficial, sendo aceita também em diversas outras farmacopeias. No Brasil é popularmente utilizada para cicatrização de feridas, queimaduras, conjuntivite, dores reumáticas dentre outros (Freitas, Rodrigues \& Gaspi, 2014).

Em relação aos mecanismos que sugerem o efeito citotóxico provocado pela Aloe vera, esses parecem depender da dose utilizada e do tipo de tumor. Sugere-se que há uma redução na proporção de células na fase mitótica por indução de apoptose provocada pelas antraquinonas. Quanto à sua toxicidade, existem relatos de hepatite aguda além de sintomas como, cólicas, náuseas e diarreia (Ramos \& Pimentel, 2011).

A Aloe vera é rica em substâncias como aloína, alantoína e antraquinonas, que são cicatrizadoras, porém, sua ingestão pode causar efeitos tóxicos. Entretanto, um estudo apontou que a toxicidade da babosa é menor que comparada à toxicidade de medicamentos, como a prednisolona (Ramos \& Pimentel, 2011). Essa espécie não deve ser ingerida por mulheres durante a menstruação, gravidez e em estados hemorroidários. Seu uso também é contra-indicado internamente em crianças (Palharin et al., 2008).

\subsection{Boldo (Peumus boldus)}

A Peumus boldus é uma espécie de planta arbórea que pertence à família Monimiaceae e é nativa do Chile. Popularmente é conhecida como boldo, sendo bastante usada como planta medicinal em especial para problemas do sistema digestório (Ruiz et al., 2008).

As propriedades farmacológicas se devem em especial ao alcalóide boldina, sendo este o principal componente do chá de boldo. Preparações a base do boldo também estão descritas em farmacopéias no Brasil, Chile e Europa, além da medicina homeopática (Ruiz et al., 2008). 
Estudos apontam que o óleo essencial das folhas do boldo apresenta atividade antibacteriana contra Streptococcus pyogenes, Micrococcus sp., Sthaphylococcus aureus, Bacilus subtilis e antifúngica contra diversas espécies de Candida. Diversos estudos apontam o boldo com excelente capacidade antioxidante devido à habilidade em seqüestrar radicais hidroxila e peroxila. Também possuindo capacidade de atenuar o desenvolvimento de diabetes e de tumores em estudos experimentais. Em amostras de sangue humano, o boldo se mostra capaz de inibir a agregação plaquetária. Outros estudos apontam atividade antiinflamatória e bloqueadora da junção neuromuscular (Ruiz et al., 2008).

A Peumus boldus na forma de chá deve ser consumida com bastante moderação e precaução, em especial durante a gravidez devido ao risco de teratogenia. Outros estudos relatam risco de dermatite alérgica, hepatotoxicidade, aumento do efeito anticoagulante em pacientes sob uso de varfarina. Na literatura existe apenas um caso comprovado de anafilaxia com o consumo do chá de boldo (Almeida et al., 2000).

Um estudo que avaliou a toxicologia do extrato hidroetanólico das folhas de $P$. boldus em ratas prenhes $(800 \mathrm{mg} / \mathrm{kg}$, via oral, dose única) foram observados efeitos teratogênicos e abortivos. Nesse mesmo estudo, realizado com ratos machos tratados por via oral com o extrato bruto do boldo, durante 90 dias, a partir do trigésimo dia já apresentaram aumento considerável de colesterol e de transaminases e uma redução dos níveis de bilirrubina total, glicose e uréia (Almeida et al., 2000).

De acordo com a literatura, a Peumus boldus, por possuir alcalóides em sua estrutura, tem seu uso recomendado a partir dos seis anos de idade, sendo contra-indicado antes disso. Uma pesquisa detectou que o óleo essencial na dose de 0,07g/k, produz convulsões em cobaias, não sendo assim indicado em pessoas com histórico de convulsões, em especial crianças (Torres et al., 2005).

\subsection{Erva doce (Pimpinella anisum)}

A Pimpinella anisum L., é uma planta da família Umbelliferae, bastante comercializada principalmente em formas de chá, sendo popularmente conhecido como erva-doce ou anis. De origem mediterrânea, e frequentemente encontrada no estado de Santa Catarina é bastante usada como antiespasmódica e inibidora da fermentação intestinal (Nascimento et al., 2005).

A erva doce é uma planta cujas folhas são utilizadas popularmente para prisão de ventre, dores estomacais, cólicas intestinais, antiespasmódica, carminativa, expectorante e regularizadora das funções menstruais. Estudos também já demonstraram atividades broncodilatadora, antioxidante e antimicrobiana, além de conseguir reduzir os efeitos causados pelo uso da morfina (Nascimento et al., 2005) (Tambosi \& Rogge-Renner, 2010).

Em relação aos efeitos tóxicos, em grande quantidade a erva doce pode causar broncodilatação, aumento da produção de estrógeno e alergias. O chá de erva doce é um dos chás mais consumidos atualmente, principalmente por pessoas que buscam sua ação tranqüilizante e/ou diurética. Entretanto, seus componentes podem, caso a planta seja consumida em excesso, possuir atividade tóxica. Como se trata de uma planta utilizada na medicina popular para terapêutica de diversas patologias ela deve ser monitorada por seus efeitos maléficos para a saúde humana (Mello, Langeloh \& Mello, 2007).

Um estudo avaliou as plantas medicinais mais utilizadas por crianças, e que se usadas de forma inadequada podem ser nocivas. Nessa pesquisa a erva doce foi a segunda mais consumida, ficando atrás apenas da camomila em quantidade de consumo. Nesse mesmo estudo, identificou-se que doses tóxicas da erva doce podem ser responsáveis por eventuais tumores e convulsões (Santos, 2014).

\subsection{Hortelã (Mentha piperita)}

A Mentha piperita Linn., é uma planta pertencente à família Lamiaceae, e encontrada principalmente em países do hemisfério Norte. Popularmente é conhecida como hortelã-pimenta e menta. Seu uso industrial se dá principalmente na área 
farmacêutica, de gomas de mascar e de bebidas alcoólicas. Suas folhas são bastante utilizadas como aromatizantes de chás e alimentos (David, Boaro \& Marques, 2006).

A Mentha piperita Linn., é conhecida popularmente por hortelã-pimenta e se caracteriza por ser uma erva perene, com caule ramificado, contendo folhas opostas pecioladas ovais. Essa planta produz mentol em grande quantidade, sendo nas folhas a maior concentração dos mesmos. O óleo essencial da hortelã-pimenta possui grande importância econômica e caracteriza por possuir propriedades antiespasmódica, antiinflamatória, antiúlcera e antiviral (Haber et al., 2015).

Algumas pesquisas indicam que o chá de hortelã é um dos mais consumidos no mundo inteiro, especialmente por suas folhas serem ricas em mentol. Estudos já demonstraram que a hortelã possui comprovadamente efeitos antimicrobianos e antivirais, atividades antioxidantes e antitumorais potentes, e um leve potencial alergênico. Outros estudos já demonstraram atividade relaxante do trato gastrointestinal, analgésica, anestésica e imunomoduladora. Ensaios clínicos já foram realizados em busca de saber o efeito da hortelã sobre a síndrome do intestino irritável, entretanto foram estudos limitados. Outros estudos contra-indicam o uso da hortelã em pacientes com refluxo gastrointestinal, hérnia hiatal ou pedras nos rins (Mckay \& Blumberg, 2006).

Poucos são os efeitos tóxicos da hortelã descritos na literatura, mas sabe-se que devido existir o mentol em sua composição em quantidade considerável, pode causar dispnéia e asfixia, especialmente em crianças e lactentes (Rodrigues et al., 2011).

\subsection{Malva Santa (Plectranthus barbatus andr.)}

O Plectranthus barbatus andr. (P. barbatus) é uma espécie pertencente à família Lamiaceae, de origem africana e encontrada em todo o Brasil sendo uma das plantas mais consumidas em nosso país. Caracteriza-se especialmente por suas propriedades analgésica e antidispéptica. Popularmente é conhecida malva santa, sete-dores, boldo nacional e tapete-de-Oxalá. Aparentemente é semelhante a hortelã da folha grande, podendo ser confundida (Costa, 2006).

A P. barbatus Andr. tem atividade terapêutica sobre mal-estar no estômago e no fígado, gastrite, auxilia na digestão e dores de barriga. Há estudos que demonstram também seu poder hiposecretor gástrico, auxiliando assim no controle da gastrite e mal-estar gástrico, na dispepsia, azia e ressaca (Costa \& Nascimento, 2003).

Estudos relatam as diversas finalidades terapêuticas difundidas em todo o mundo dos extratos de P. barbatus. Os mesmos têm sido aplicados para tratamentos cardiovasculares, respiratórios, gastrointestinais e do sistema nervoso central. Constituintes da planta possuem efeito inotrópico positivo, hipotensivo e potente inibidor da agregação plaquetária (Costa, 2006).

Quanto à toxicologia, sabe-se que pode ser fatal para o feto e ser utilizado para interromper a gravidez. Um estudo do extrato de P. barbatus em cobaias mostrou retardamento no desenvolvimento fetal e um efeito anti-implantação, reafirmando assim seu potencial abortivo. Pesquisadores revelam que o uso prolongado da planta, como é comum especialmente em comunidades carentes, é de grande preocupação podendo levar o paciente a apresentar problemas hepatotóxicos, carcinogênicos e nefrotóxicos em casos de consumo a longo prazo. Por se caracterizar em ter seus efeitos como assintomáticos, o paciente pode demorar a perceber o mal que a planta está fazendo (Costa, 2006).

\subsection{Manjericão (Ocimum basilicum)}

A Ocimum basilicum L., planta pertencente à família Lamiaceae e é conhecida popularmente como manjericão. Originário da Índia, tem seu uso bastante difundido em especial nos Estados Unidos como planta aromatizante, e bastante importante para a economia de plantas medicinais (Luz et al., 2014). 
O óleo essencial das folhas de manjericão também apresenta propriedades inseticidas e repelentes. Estudos in vitro apontam que o óleo de manjericão é capaz de inibir mediadores envolvidos no processo de inflamação (Machado et al., 2011).

Devido o manjericão ser uma planta com diversos compostos químicos que possuem potencial terapêutico na redução da glicemia, redução de lipídeos plasmáticos, podendo ser assim uma forma de prevenção de doenças como o diabetes e as cardiovasculares. Estudos em cobaias demonstraram grande redução nos valores de triglicerídeos, colesterol total e glicemia dos grupos tratados com o Ocimum basilicum L. e aumento significativo nos níveis de HDL (Machado et al., 2011).

O manjericão possui em sua composição a cânfora e pinocanfona que são neurotóxicos em altas doses de consumo da planta, nesses casos podendo assim provocar convulsões, distúrbios sensoriais e até psíquicos. $\mathrm{O}$ uso do manjericão é contraindicado em gravidez, pois possui atividades anticonceptivas (Cavalini et al., 2005).

\subsection{Mastruz (Chenopodium ambrosioides)}

A espécie Chenopodium ambrosioides $l$. é uma planta da família Chenopodiaceae que apresenta ampla distribuição no mundo inteiro, sendo uma das espécies mais utilizadas na medicina popular. Popularmente é conhecida como erva de santa maria, mastruz ou mastruço, sendo bastante indicadas para tratamento de feridas, inflamações da pele, contusões e fraturas (Trindade et al., 2015).

A Chenopodium ambrosioides l., é uma planta medicinal herbácea, se caracteriza pelo forte odor, de origem da América do Sul, que ocorre em todo o Brasil, sendo considerada como planta daninha em algumas regiões do Brasil. As folhas e frutos acumulam óleo essencial rico em ascaridol, princípio ativo responsável pelo efeito vermífugo da planta. O mastruz já era muito utilizado principalmente pelas civilizações indígenas da América do Norte e do Sul, destacando-se o uso para combater a disenteria e picadas de insetos e aranhas (Trindade et al., 2015).

Atualmente o mastruz é bastante utilizado contra pneumonia, tuberculose e rouquidão, devido suas ações como expectorante e antitussígeno. Seu uso como anti-helmíntico é bastante consagrado também (Souza \& Ribeiro, 2008).

Em um estudo que objetivou avaliar o dano genético do extrato de mastruz em linfócitos humanos, constatou que o extrato causou aberrações cromossômicas, mudou a velocidade de proliferação celular e índices mitóticos (Galdiano et al., 2007). Outra pesquisa sobre a ação toxicológica do mastruz verificou que na concentração de $50 \mathrm{mg} / \mathrm{kg}$ o extrato induziu sinais de toxicidade renal e na dose de 500mg/kg induziu alterações de comportamento e fisiológicas, além de sinais de hepatotoxicidade (Pereira, 2010).

\subsection{Quebra pedra (Phyllanthus sp.)}

O termo quebra pedra é utilizado popularmente para designar diversas espécies de um mesmo gênero (Phyllanthus) que ocorre amplamente nas regiões tropicais e é encontrado em todos os estados brasileiros. Entre as espécies do gênero podese citar Phyllanthus Niruri L., Phyllanthus Tenellus Roxb, Phyllanthus Amarus Schumach, entre outras. No Brasil, as folhas de quebra-pedra são bastante utilizadas na forma de infusão, já em regiões orientais, é indicado o uso das raízes de quebra-pedra para aplicações tópicas (Mellinger, 2006).

As diversas espécies definidas como quebra-pedra são utilizadas na medicina popular para a terapêutica de cálculo renal, urinário, antiespasmódicas e no controle da diabetes por serem hipoglicemiantes. Estudos já realizados com esse gênero demonstraram também atividades antihepatotóxica, diurética, reguladora estomacal, antiinfecciosa em relação ao sistema urinário, etc. (Nascimento et al., 2005).

O uso de quebra-pedra pode ser tóxico, pois na sua composição possui alcalóides com potencial de toxicidade e tem contra-indicação por períodos prolongados e de forma alguma durante o período de gravidez e amamentação, pois a mesma 
possui princípios que atravessam a barreira placentária, e pode induzir o aborto, além de ser excretadas no leite materno (Rodrigues et al., 2005).

\subsection{Pata de Vaca (Bauhinia forficata l.)}

A Bauhinia forficata L. é uma planta pertencente à família Leguminosae. Popularmente é conhecida como pata-devaca ou unha-de-vaca, sendo amplamente utilizada como diurética no tratamento de diabetes (Miyake, Akisue \& Akisue, 1986).

Essa espécie se caracteriza por suas atividades farmacológicas, possuindo ação hipoglicemiante e diurética. Também relata-se uma sutil atividade antiinflamatória e analgésica (Oliveira et al., 1999).

A literatura aponta que apesar de serem diversos os estudos farmacológicos que apresentam resultados relevantes com a Bauhinia forficata L., especialmente em relação à diabetes, a avaliação da toxicidade tem sido pouco investigada. Uma pesquisa caracterizou e avaliou a toxicidade do extrato seco da pata-de-vaca padronizado para o desenvolvimento de formas farmacêuticas sólidas. A avaliação toxicológica foi realizada através do estudo agudo e subcrônico. Verificou-se aumento dos níveis de ALT e fosfatase alcalina e a indicação de uma possível toxicidade hepática (Peixoto et al., 2012). Em outro estudo, os pesquisadores avaliaram diferentes concentrações do extrato etanólico da pata-de-vaca. Entre as três concentrações testadas, o extrato que apresentou maior CL50 foi o de $1000 \mu \mathrm{g} / \mathrm{mL}$ sendo considerado o menos tóxico, enquanto que o extrato que apresentou CL50 menor que $500 \mu \mathrm{g} / \mathrm{mL}$ foi considerado altamente tóxico (Martínez et al., 2011). Entretanto, outra pesquisa teve resultados que não indicaram atividade mutagênica e citotóxica em células sanguíneas (Manso, 2014).

\section{Conclusão}

É indiscutível tanto a importância econômica quanto o potencial terapêutico de plantas medicinais no Brasil. Esse potencial é associado ao conhecimento tradicional das plantas e dos seus efeitos tóxicos, além de uma correta orientação profissional pode ser utilizado de forma a garantir a eficácia, segurança e qualidade dos medicamentos fitoterápicos. As plantas medicinais são muito utilizadas no tratamento de doenças, entretanto seus efeitos tóxicos na maioria das vezes são desconhecidos pela população e muitas vezes até pelos profissionais de saúde. Entre os efeitos tóxicos mais encontrados destacou-se teratogenia, abortos, citotoxicidade e hepatotoxicidade. Diante do exposto, é necessário que além dos estudos farmacológicos, sejam realizados mais estudos toxicológicos das plantas com potencial farmacêutico, para assim viabilizar mais ainda seu uso, inserindo mais opções terapêuticas para a população.

\section{Referências}

Almeida, E. R. D., Melo, A. M., \& Xavier, H. (2000). Toxicological evaluation of the hydro-alcohol extract of the dry leaves of Peumus boldus and boldine in rats. Phytotherapy Research: An International Journal Devoted to Pharmacological and Toxicological Evaluation of Natural Product Derivatives, 14(2), 99102. https://doi.org/10.1002/(SICI)1099-1573(200003)14:2<99::AID-PTR600>3.0.CO;2-4

Argenta, S. C., Argenta, L. C., Giacomelli, S. R., \& Cezarotto, V. S. (2011). Plantas medicinais: cultura popular versus ciência. Vivências, 7(12), 51-60. http://www2.reitoria.uri.br/ vivencias/Numero_012/artigos/artigos_vivencias_12/n12_05.pdf

Barreiro, E. J., \& Bolzani, V. D. S. (2009). Biodiversidade: fonte potencial para a descoberta de fármacos. Química nova, 679-688. https://repositorio.unesp.br/handle/11449/26002

Bochner, R., Fiszon, J. T., Assis, M. A., \& Avelar, K. E. S. (2012). Problemas associados ao uso de plantas medicinais comercializadas no Mercadão de Madureira, município do Rio de Janeiro, Brasil. Revista Brasileira de Plantas Medicinais, 14(3), 537-547. http://dx.doi.org/10.1590/S151605722012000300017

Cavalini, M., Folis, G. P., Resener, M. C., Alexandre, R. F., Zannin, M., \& Simões, C. M. O. (2005). Serviço de informações sobre plantas medicinais e medicamentos fitoterápicos. Extensio: Revista Eletrônica de Extensão, 2(2):1-11. https://periodicos.ufsc.br/index.php/extensio/article/view/5131

Costa, M. C. C. D. (2006). Uso popular e ações farmacológicas de Plectranthus barbatus Andr.(Lamiaceae): revisão dos trabalhos publicados de 1970 a 2003. Revista Brasileira de Plantas Medicinais, 8(2), 81-88. https://www1.ibb.unesp.br/Home/Departamentos/Botanica/RBPMRevistaBrasileiradePlantasMedicinais/revisao.pdf 
Costa, M. C. C. D., \& Nascimento, S. C. (2003). Atividade citotóxica de Plectranthus barbatus Andr.(Lamiaceae). Acta Farm. Bonaerense, 22, 155-158. http://www.latamjpharm.org/trabajos/22/2/LAJOP_22_2_1_9_RNLD26JA96.pdf

Cunha, G. H. D. (2012). Efeito farmacológico das frações hexânica, clorofórmica e metanólica do óleo essencial da Alpinia zerumbet na reatividade vascular in vitro e nos parâmetros cardiovasculares in vivo. http://repositorio.ufc.br/handle/riufc/5554

David, E. F. S., \& Boaro, C. S. F. (2009). Translocação orgânica, produtividade e rendimento de óleo essencial de Mentha piperita L. cultivada em solução nutritiva com variação dos níveis de N, P, K e Mg. Revista Brasileira de Plantas Medicinais, 236-246. https://repositorio.unesp.br/handle/11449/5243 de Paula Ramos, A., \& Pimentel, L. C. (2011). Ação da Babosa no reparo tecidual e cicatrização. Brazilian Journal of Health, 2(1), 40-48. https://pielsana.cdn.prismic.io/pielsana/e1069baa-6d14-48ee-80f0-

eb1772adec79_A\%C3\%A7\%C3\%A3o+da+Babosa+no+reparo+tecidual+e+cicatriza\%C3\%A7\%C3\%A3o.pdf

Freitas, V. S., Rodrigues, R. A. F., \& Gaspi, F. O. G. (2014). Propriedades farmacológicas da Aloe vera (L.) Burm. f. Revista brasileira de plantas medicinais, 16(2), 299-307. http://dx.doi.org/10.1590/S1516-05722014000200020

Galdiano, L. C., Chicone, A. C. V., Nunes, L. G., Freitas, M. D. P. N., \& Iamagutti, P. S. (2007). Levantamento das plantas medicinais cultivadas e comercializadas por horticultores no município de ituverava-SP. Nucleus, 4(1), 1-4. https://dialnet.unirioja.es/servlet/articulo?codigo=4032307

Gorberg W., \& Fridman, H. B. (2006). Chás miraculosos. Revista Saúde: Especial Bem-Estar. Azul.

Haber, L. L., Luz, J. M. Q., Arvati Dóro, L. F., \& Santos, J. E. (2005). Diferentes concentrações de solução nutritiva para o cultivo de Mentha piperita e Melissa officinalis. Horticultura Brasileira, 23(4), 1006-1009. https://doi.org/10.1590/S0102-05362005000400029

Luz, J. M. Q., de Resende, R. F., Silva, S. M., De Santana, D. G., da S Camilo, J., Blank, A. F., \& Haber, L. L. (2014). Produção de óleo essencial de Ocimum basilicum L. em diferentes épocas, sistemas de cultivo e adubações. Boletín Latinoamericano y del Caribe de Plantas Medicinales y Aromáticas, 13(1), 69-80. https://www.redalyc.org/pdf/856/85629766007.pdf

Machado, F. M. V. F., Barbalho, S. M., da Silva, T. H. P., dos Santos Rodrigues, J., Guiguer, E. L., dos Santos Bue no, P. C., \& Araújo, A. C. (2011). Efeitos do uso de manjericão (Ocimum basilicum L.) no perfil bioquímico de ratos Wistar. J Health Sci Inst, 29(3), 191-194.

Manso, J. A. X., Hanusch, A. L., da Silva, C. C., da Cruz, A. D., \& da Cruz, D. M. (2015). Avaliação do Potencial Mutagênico de Bauhinia L. SP. Revista EVS-Revista de Ciências Ambientais e Saúde, 41, 75-82. http://dx.doi.org/10.18224/est.v41i0.3853

Martínez, M. M., Ocampo, D. M., Galvis, J. H., \& Valencia, A. (2011). Antibacterial activity and in vivo cytotoxicity of ethanol extracts from Bauhinia

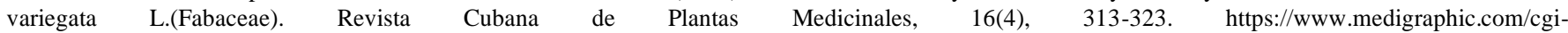
bin/new/resumenI.cgi?IDREVISTA=262\&IDARTICULO=36091\&IDPUBLICACION=3835

Matos, F. J. A. (1998). Farmácias vivas: Editora da Universidade Federal do Ceará.

McKay, D. L., \& Blumberg, J. B. (2006). A review of the bioactivity and potential health benefits of chamomile tea (Matricaria recutita L.). Phytotherapy Research: An International Journal Devoted to Pharmacological and Toxicological Evaluation of Natural Product Derivatives, 20(7), 519530.https://doi.org/10.1002/ptr.1900

Mellinger, C. G. (2006). Caracterização estrutural e atividade biológica de carboidratos de Phyllanthus niruri (Quebra-pedra). https://www.acervodigital.ufpr.br/handle/1884/8029

Mello, F. D., Langeloh, A., \& Mello, A. B. (2007). Estudo de toxicidade pré-clínica de Fitoterápico contendo Pimpinella anisum, Foeniculum foeniculum, Sambucus australis e Cassia augustifolia. Lat Am J Pharm, 26, 230-237. http://www.latamjpharm.org/trabajos/26/2/LAJOP_26_2_1_10_86954LT6H6.pdf

Mena-Huertas, S. J., García-López, J. P., Nicola-Benavides, S. N., \& Yépez-Chamorro, M. C. (2016). Inocuidad citotóxica y mutagénica de los aceites esenciales de Rosmarinus officinalis. L y Ruta graveolens L. promisorios para el tratamiento complementario de la infección por Helicobacter pylori. Actualidades Biológicas, 38(104), 37-44. https://revistas.udea.edu.co/index.php/actbio/article/view/331463

Miyake, E. T., Akisue, G., \& Akisue, M. K. (1986). Caracterização farmacognóstica da pata-de-vaca Bauhinia forficata Link. Revista Brasileira de Farmacognosia, 1(1), 58-68. https://doi.org/10.1590/S0102-695X1986000100007.

Nascimento, P. F., Nascimento, A. C., Rodrigues, C. S., Antoniolli, Â. R., Santos, P. O., Barbosa Júnior, A. M., \& Trindade, R. C. (2007). Atividade antimicrobiana dos óleos essenciais: uma abordagem multifatorial dos métodos. Revista Brasileira de Farmacognosia, 17(1), 108113.http://dx.doi.org/10.1590/S0102-695X2007000100020

Nascimento, V. T., Lacerda, E. U., Melo, J. G., Lima, C. S. A., Amorim, E. L. C., \& Albuquerque, U. P. (2005). Controle de qualidade de produtos à base de plantas medicinais comercializados na cidade do Recife-PE: erva-doce (Pimpinella anisum L.), quebra-pedra (Phyllanthus spp.), espinheira santa (Maytenus ilicifolia Mart.) e camomila (Matricaria recutita L.). Revista Brasileira de Plantas Medicinais, 7(3), 56-64. https://www.researchgate.net/profile/Ulysses_Albuquerque/publication/287938747_

Oliveira, F. D., Kato, E. T. M., Rodrigues, R. F. D. O., \& Basso, S. L. (2001). Mitos e verdades sobre pata-de-vaca-Bauhinia forficata Link-uma revisão. Lecta-USF, 19(1/2), 7-20. https://pesquisa.bvsalud.org/portal/resource/pt/lil-313474

Palharin, L. H. D. C., Figueiredo Neto, E., Camargo-Lopes, M. P., \& Bosquê, G. G. (2008). Efeitos fitoterápicos e homeopáticos da babosa. Revista Científica Eletrônica de Agronomia, 7(14),1-6. ttp://faef.revista.inf.br/imagens_arquivos/arquivos_destaque/U71PdgToK70xtc4_2013-5-10-12-16-59.pdf

Peixoto Sobrinho, T. J. S., Gomes, T. L. B., Cardoso, K. C. M., Albuquerque, U. P., \& Amorim, E. L. C. (2012). Teor de flavonóides totais em produtos contendo pata-de-vaca (Bauhinia L.) comercializados em farmácias de Recife/PE. Revista Brasileira de Plantas Medicinais, 14(4), 586-591. https://doi.org/10.1590/S1516-05722012000400003 
Research, Society and Development, v. 10, n. 5, e55910514821, 2021

(CC BY 4.0) | ISSN 2525-3409 | DOI: hittp://dx.doi.org/10.33448/rsd-v10i5.14821

Pereira, W. S., Ribeiro, B. P., Sousa, A. I., Serra, I. C., Mattar, N. S., Fortes, T. S., \& Nascimento, F. R. (2010). Evaluation of the subchronic toxicity of oral treatment with Chenopodium ambrosioides in mice. Journal of ethnopharmacology, 127(3), 602-605. https://doi.org/10.1016/j.jep.2009.12.018

Prashar, A., Hili, P., Veness, R. G., \& Evans, C. S. (2003). Antimicrobial action of palmarosa oil (Cymbopogon martinii) on Saccharomyces cerevisiae. Phytochemistry, 63(5), 569-575. https://doi.org/10.1016/S0031-9422(03)00226-7

Reis, K. B., Côrtes, M. V. D. C. B., Martins, F. S., Filippi, M. C. C. D., Paula, J. R. D., \& Conceição, E. C. D. (2015). Characterization of rue extract and its potential for controlling rice blast. Pesquisa Agropecuária Brasileira, 50(12), 1121-1130.https://doi.org/10.1590/S0100-204X2015001200001

Rodrigues, H. G., Meireles, C. G., Lima, J. T. S., Toledo, G. P., Cardoso, J. L., \& Gomes, S. L. (2011). Efeito embriotóxico, teratogênico e abortivo de plantas medicinais. Revista brasileira de plantas medicinais, 13(3), 359-366. https://doi.org/10.1590/S1516-05722011000300016

Ruiz, A. L. T., Taffarello, D., Souza, V. H., \& Carvalho, J. E. (2008). Farmacologia e toxicologia de Peumus boldus e Baccharis genistelloides. Revista Brasileira de Farmacognosia, 18(2), 295-300. http://dx.doi.org/10.1590/S0102-695X2008000200025

Santos, A. A. O uso de fitoterápicos e plantas medicinais no cuidado de crianças: O papel do enfermeiro [monografia]: Centro de ciências biológicas e da saúde/ Universidade Estadual da Paraíba; 2014. 26p.

Souza, A. D., \& Ribeiro, V. V. (2008). Perfil dos raizeiros e estudos de suas indicações acerca das plantas medicinais utilizadas no tratamento das doenças do trato respiratório. Revista de Biologia e Farmácia, 3(1), 102-109.

Tambosi, G., \& Rogge-Renner, G. D. (2010). Avaliação de métodos de esterilização, concentração de ágar e composição de meio de cultura para propagação in vitro de Pimpinella anisum (Linn.)-Apiaceae. Semina: Ciências Biológicas e da Saúde, 31(2), 189-194. http://www.uel.br/revistas/uel/index.php/seminabio/article/view/8798

Tôrres, A. R., Oliveira, R. A. G. D., Diniz, M. D. F. F. M., \& Araújo, E. C. (2005). Estudo sobre o uso de plantas medicinais em crianças hospitalizadas da cidade de João Pessoa: riscos e benefícios. Revista Brasileira de Farmacognosia, 15(4), 373-380. http://dx.doi.org/10.1590/S0102-695X2005000400019

Trindade, R. C. P., Ferreira, E. S., Gomes, I. B., Silva, L., Sant'ana, A. E. G., Broglio, S. M. F., \& Silva, M. (2015). Extratos aquosos de inhame (Dioscorea rotundata Poirr.) e de mastruz (Chenopodium ambrosioides L.) no desenvolvimento da lagarta-do-cartucho-do-milho Spodoptera frugiperda (JE Smith, 1797). Revista Brasileira de Plantas Medicinais, 17(2), 291-296. https://doi.org/10.1590/1983-084X/13_082

Valadas, L. A. R., Lobo, P. L. D., Fonseca, S. G. D. C., Fechine, F. V., Rodrigues Neto, E. M., Fonteles, M. M. D. F. \& Bandeira, M. A. M. (2021). Clinical and Antimicrobial Evaluation of Copaifera langsdorffii Desf. Dental Varnish in Children: A Clinical Study. Evidence-Based Complementary and Alternative Medicine, 2021, ID 6647849 . 